\title{
Urgent simultaneous revascularization of the carotid artery and ascending aortic replacement for type $A$ acute aortic dissection with cerebral malperfusion
}

\author{
Keishi Ueyama, MD, PhD · Kennji Otaki, MD \\ Makoto Koyama, MD · Hiroyasu Kamiyama, MD
}

Received: 22 December 2006 / Accepted: 13 April 2007

(C) The Japanese Association for Thoracic Surgery 2007

\begin{abstract}
We report a case of successful urgent surgical therapy for a Stanford type A acute aortic dissection with cerebral malperfusion. A woman with a sudden severe chest pain consulted the emergency department. Computed tomography results showed a Stanford type A acute aortic dissection. She had repeating left paralysis, so a cerebrovascular study was performed. It showed that the collateral left carotid and vertebral arteries perfused only the right cerebral hemisphere. Hence, it was thought that the left paralysis was caused by cerebral malperfusion due to the dissection. Simple aortic replacement endangered the right cerebral hemisphere, so we performed urgent revascularization of the right carotid artery, followed by an ascending aortic replacement procedure.
\end{abstract}

Key words Aortic dissection - Cerebral complications · Endarterectomy $\cdot$ Malperfusion

\section{Introduction}

A Stanford type A acute aortic dissection usually requires urgent surgery. However, in patients with cerebral malperfusion, simple surgical aortic replacement to close the

K. Ueyama $(\bowtie) \cdot$ K. Otaki $\cdot$ M. Koyama

Department of Cardiovascular Surgery, Asahikawa Red Cross

Hospital, Akebono 1-1-1-1, Asahikawa 070-8530, Japan

Tel. +81-166-22-8111; Fax +81-166-24-4648

e-mail: kueyama@asahikawa-rch.gr.jp

H. Kamiyama

Department of Neurosurgery, Asahikawa Red Cross Hospital,

Asahikawa, Japan entry of the dissection may cause cerebral infarction. Thus, conservative therapy is generally the first choice in those cases, ${ }^{1}$ although it carries some risk of mortality. We report a successful urgent simultaneous revascularization of the right carotid artery and ascending aortic replacement for a case of type A acute aortic dissection with cerebral malperfusion.

\section{Case}

A 72-year-old woman experienced a sudden onset of severe chest pain and came to the emergency department of our hospital by ambulance. Emergent enhanced chest computed tomography (CT) results showed a Stanford type A acute aortic dissection. After use of an analgesic and depressor, clouding of consciousness and left paralysis appeared, which lessened for a few minutes but reappeared when the blood pressure was reduced to less than $100 \mathrm{mmHg}$. Ultrasonography findings showed a dissection of the right common carotid artery; thus, urgent magnetic resonance imaging (MRI)-CT of the brain, single photon emission computed tomography (SPECT) of the brain, and enhanced three-dimensional (3D)-CT angiography of the neck were performed. The brain MRI-CT showed no cerebral infarction. However, SPECT revealed diminished perfusion on the right side of the brain (Fig. 1), and 3D-CT angiography of the neck showed occlusion of the right internal carotid artery. Therefore, we believed that the right internal carotid artery was occluded by malperfusion due to the dissection, and the collateral left carotid and vertebral arteries supplied perfusion to the right brain only. We concluded that when blood pressure was reduced perfusion of the right brain was diminished and the symptoms 


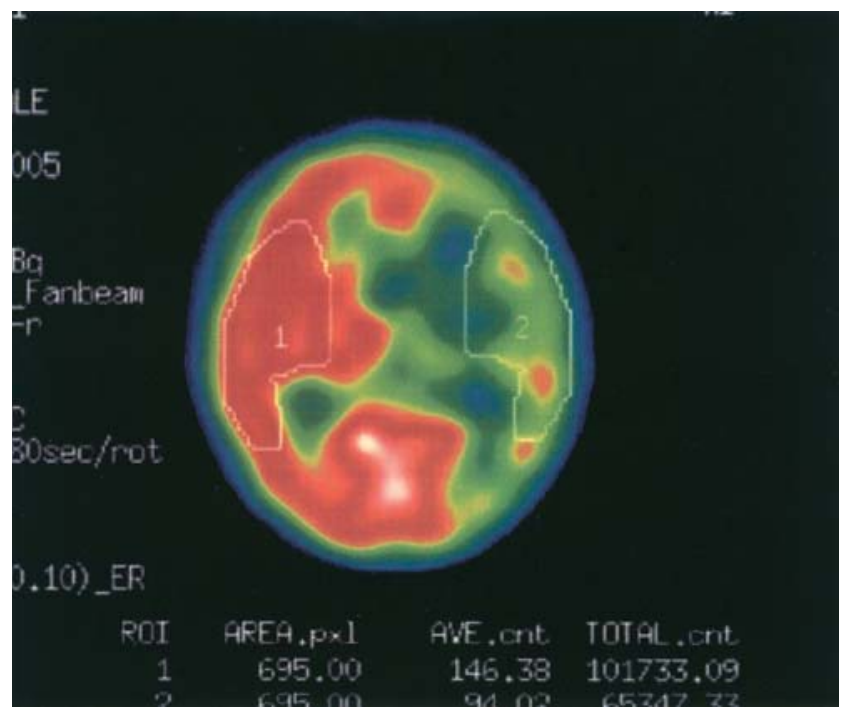

Fig. 1 Single photon emission computed tomography (SPECT) of the brain revealed diminished perfusion in the right hemisphere, especially the right middle cerebral artery perfusion area

appeared. The right brain was thus at risk for cerebral infarction.

If aortic replacement alone was performed, the blood pressure would be reduced during extracorporeal circulation, with some risk of left hemiplegia. Therefore, we decided to perform cerebrovascular reperfusion first, following aortic replacement. Together, these examinations and diagnosis required $5 \mathrm{~h}$; the total time from onset to surgery was $7 \mathrm{~h}$.

Urgent surgery was performed under general anesthesia, and the right carotid artery was opened by a neurosurgeon. The common carotid artery and internal carotid artery had been dissected, and a thrombus was found in a false lumen. In contrast, there was no thrombus in the true lumen, although it seemed to be compressed (Fig. 2). Perfusion of the internal carotid artery was maintained by use of a temporary shunt. The intimal flaps at the internal, external, and common carotid arteries were resected. This procedure was similar to carotid endarterectomy. Next, a vertebral artery-to-internal carotid artery bypass was performed using the right radial artery. Thereafter, the neck incision was left open, and ascending aortic replacement surgery was started.

A midline skin incision of the chest and a full sternotomy were performed; some thin, bloody pericardial effusion was seen. An extracorporeal circulation was established with a right femoral arterial inlet, along with superior vena cava and inferior vena cava drainage. Under profound hypothermia and retrograde cerebral perfusion, the ascending aorta was opened and the entry of the dissection appeared on the right side of the ascend-

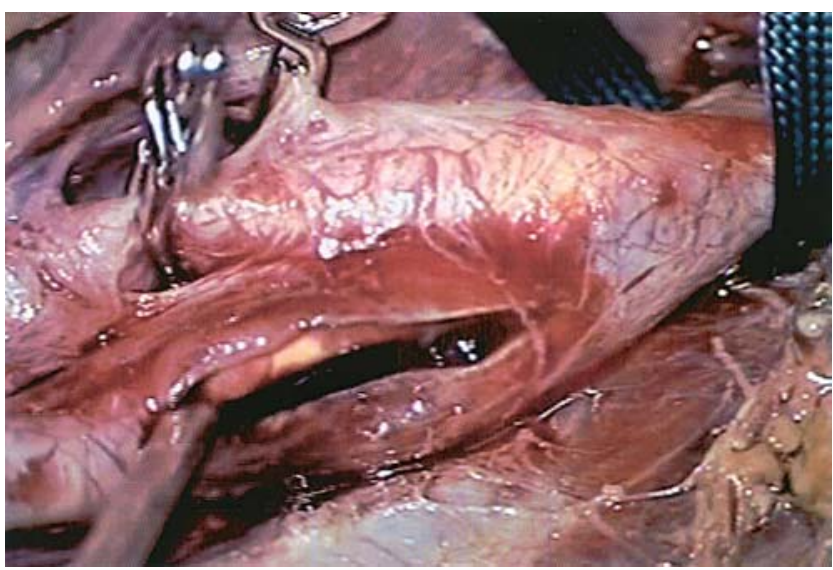

Fig. 2 View of the common carotid artery. Thrombus was found in a false lumen of the common carotid artery. In contrast, there was no thrombus in the true lumen

ing aorta. The ascending aorta was replaced with a 24mm Dacron graft (Gelsoft; Vascutek, Renfrewshire, Scotland, UK). Following thoracic surgery, we confirmed that there was no bleeding in the neck surgical area, and closure was performed. The total operating time was $12 \mathrm{~h} 15 \mathrm{~min}$, with a thoracic operating time of $4 \mathrm{~h} 20 \mathrm{~min}$.

The patient was weaned from the ventilator on the second postoperative day, and she was discharged from the intensive care unit on day 5. Thereafter, the patient recovered smoothly without any palsy or complications, and cerebral CT and MRI scans showed no infarction or other changes. Furthermore, the 3D-CT results showed that a pseudo-lumen of the carotid artery and aortic arch partially remained enhanced from the distal anastomosis of the aorta to the surgical portion of the right carotid artery. The pseudo-lumen, from the arch to the descending aorta, was not enhanced. In the common carotid artery the aortic septum had disappeared, which was thought to be due to the endarterectomy. MR angiography showed good cerebral perfusion. The patient was discharged from the hospital on postoperative day 33 .

\section{Discussion}

Among patients with a Stanford type A acute aortic dissection, $5 \%-15 \%$ of the cases are complicated by dissection of the carotid artery, ${ }^{2,3}$ and $5 \%-10 \%$ have cerebrovascular complications., ${ }^{2,4}$ Dissection of the arch branches is more likely to occur with the brachiocephalic artery than the left carotid or left subclavian arteries because dissection of the ascending aorta can easily progress to the brachiocephalic artery; thus left-side 
hemiplegia is more common..$^{2-4}$ In this case, intermittent left-side paralysis occurred because of malperfusion of the right carotid artery.

Urgent surgery is usually required for salvage in patients with Stanford type A acute aortic dissection. However, the operative prognosis for patients with cerebral malperfusion is poor. ${ }^{1-4}$ Thus, some have reported that operative treatment is relatively contraindicated. ${ }^{1-4}$ In our case, the right carotid artery was completely occluded, and the right brain was jeopardized. Furthermore, blood flow in the right cerebral hemisphere was supplied collaterally from the left carotid and vertebral arteries. When blood pressure was reduced to less than $100 \mathrm{mmHg}$, left paralysis appeared. During surgery, the extracorporeal circulation blood pressure must be reduced to lower than $100 \mathrm{mmHg}$, and the right cerebral hemisphere is likely to be damaged. Thus, we thought that our patient might develop permanent hemiplegia from that condition and decided to perform revascularization of the right carotid artery first. A number of examinations were done prior to surgery that required an extensive period of time, but they were required for the patient's safety.

\section{Conclusion}

We concluded that if the condition is stable and there are some problems with the brain in a patient with Stanford type A acute aortic dissection, cerebrovascular studies should be done before surgery. Cerebrovascular revascularization should also be considered before surgery for dissection.

\section{References}

1. Tanaka H, Okada K, Yamashita T, Morimoto Y, Kawanishi Y, Okita Y. Surgical results of acute aortic dissection complicated with cerebral malperfusion. Ann Thorac Surg 2005;80: 72-6.

2. Cambria RC, Brewster DC, Gertlet J, Moncure AC, Gusberg $\mathrm{R}$, Tilson D, et al. Vascular complication associated with spontaneous aortic dissection. J Vasc Surg 1988;7:199-209.

3. Carrel T, Laske A, Jenny B, von Segesser L, Turina M. Neurological complications associated with acute aortic dissection: is there a place for a surgical approach? Cerebrovasc Dis 1991;1:296-301.

4. Fann JI, Sarris GE, Miller DC, Mitchell RS, Oyer PE, Stinson EB, et al. Surgical management of acute aortic dissection complicated by stroke. Circulation 1989;80(suppl I):I-257-63. 